\title{
Ease of ProSeal laryngeal mask airway insertion in children (after placement) using suction catheter: A comparison with conventional techniques
}

\author{
Lalit Gupta $^{1}$, Bhavna Gupta ${ }^{2, *}$, Poonam Bhadoria ${ }^{3}$, U. C. Verma ${ }^{4}$ \\ ${ }^{1}$ Assistant Professor, ${ }^{2}$ Senior Resident, ${ }^{3,4}$ Director Professor, Dept. of Anaesthesia, Maulana Azad Medical College \& Lok Nayak \\ Hospital, New Delhi, India
}

*Corresponding Author:

Email: bhavna.kakkar@gmail.com

Received: $22^{\text {nd }}$ September, 2017

Accepted: $29^{\text {th }}$ September, 2017

\begin{abstract}
Objectives: The ProSeal Laryngeal Mask Airway (PLMA) mostly inserted by finger technique and by an introducer tool method. Recently a newer Suction Catheter Guided (SC) insertion technique has been successfully used in children. We performed a study in children with aims and objective to compare the ease of PLMA insertion using SC and conventional techniques.

Methods: 126 American Society of Anaesthesiologist's class I and II children in age group of 1-12 years weighing 10-50 kg, admitted in our hospital, undergoing an elective surgery in under general anaesthesia in supine position were included in our study. An appropriate size PLMA was inserted using Suction Catheter, introducer tool or digital technique in Groups SC, I and D respectively $(n=42)$ after induction of general anaesthesia. Correct placement of the PLMA was confirmed by using appropriate clinical tests. Ease of PLMA insertion with hemodynamic response was done by time to insertion, number of insertion attempts to achieve effective ventilation and number of cases which required lateral approach of insertion.

Results: In Group SC, PLMA device was inserted after a single attempt in 41 (97.6\%) patients, but PLMA insertion was failed in 5 patients in Group I (11.9\%) and 6 patients in Group D (14.3\%) even after two attempts, and the difference was statistically significant $(\mathrm{p}=0.044)$. Time taken for successful placement was statistically shorter in the SC-guided group (SC $22.5 \pm 4.021$ seconds, I $31.4 \pm 17.57$ seconds, D $27.10 \pm 12.9$ seconds, p value <0.05). Incidence of trauma was also less in the SC-guided group ( $\mathrm{p}$ value $<0.05$ )

Conclusion: Suction Catheter guided ProSeal LMA insertion was an easier, faster, more successful and a relatively atraumatic technique which also ensures correct placement of the PLMA in the hypopharynx as compared to the routine conventional PLMA insertion techniques with no hemodynamic pressor response to PLMA insertion using any of the three techniques.
\end{abstract}

Keywords: ProSeal LMA, Suction Catheter insertion.

\section{Introduction}

Supraglottic devices are an alternative to endotracheal tube for maintaining the airway and classic LMA is considered as gold standard of supraglottic devices. The ProSeal Laryngeal Mask Airway (PLMA) has a modified cuff and a drain tube, increased depth of bowl, which helps improve the seal with pharynx and prevents the risk of aspiration. It is routinely inserted using a digital technique similar to that of insertion of classic laryngeal mask airway (LMA) or inserted with the help of an introducer tool similar to insertion of intubating LMA. At many times, insertion of a finger in the patient's mouth may not be sufficient to place the device in correct position (especially in children) and at other times, an introducer tool may not always ensure correct placement of device in patients with different anatomy that varies from the norm. However, insertion methods of PLMA in children $^{1,2}$ are same as in adults ${ }^{2,3}$ with comparable success and failure rates. ${ }^{4}$

In literature, insertion of ProSeal LMA using Gum Elastic Bougie (GEB) has been described both in adults $^{5,6}$ and children ${ }^{7}$ with higher success rates. The disadvantages of the GEB guided technique are mainly because of stiff GEB which can cause trauma to pharyngeal and oesophageal tissues, and also it is not freely available all the times and is a costly device.

Recently, a newer method, Suction Catheter (SC) guided insertion of ProSeal LMA has been described. ${ }^{8}$ This technique avoids impaction of tip of PLMA at back of patient's mouth, which is the main cause of failure of PLMA insertion. Successful placement of PLMA using suction catheter can be also checked by using various clinical tests as with other conventional techniques. In comparison with GEB, Suction catheter is freely available in all sizes and is an economical alternative also.

Little information is available in literature about ProSeal LMA insertion in children with Suction Catheter guided technique. Hence, we found it worthy to assess the success rate of insertion and hemodynamic responses in children to PLMA placement by Suction Catheter (SC) guided technique and to compare the same using with conventional techniques.

\section{Materials and Methods}

After getting approval from our Institutional Review Board cum ethical committee board (F.No./10/IEC/MAMC/2007), this prospective randomised study was conducted in 126 American 
Society of Anesthesiologists class I and II children in age group of 1-12 years weighing $10-50 \mathrm{~kg}$, admitted in our hospital, undergoing an elective surgery in under general anaesthesia in supine position.

We excluded children who had pathology of neck, airway or gastrointestinal tract, who were at high risk of aspiration or had cardiovascular disease, bleeding disorders or patients who had history of previous difficult intubation.

After doing a thorough pre-operative anaesthetic check-up, an informed written consent was taken from parents or guardians of children who were enrolled in our study. All patients were kept fasted for 6 hour for solids and 4 hour for liquids. Premedication of all kids was done with syrup promethazine $0.5 \mathrm{mgkg}^{-1}$ orally 1 hour before induction of anaesthesia. Inside the operation theatre, all routine monitors were applied. Baseline pulse rate (PR), systolic blood pressure (SBP), diastolic blood pressure (DBP), mean blood pressure (MBP) and peripheral oxygen saturation $\left(\mathrm{SpO}_{2}\right)$ were recorded. A standard anaesthesia protocol was followed. All patients were given intravenous (iv) glycopyrollate bromide $0.01 \mathrm{mg} / \mathrm{kg}$ and they were preoxygenated with $100 \%$ oxygen for 3 minutes. General Anaesthesia was induced with fentanyl citrate $2 \mu \mathrm{g} \mathrm{kg}^{-1}$ and propofol $2 \mathrm{mg} \mathrm{kg}^{-1}$ given over 60 seconds. Child was ventilated via Bag and mask ventilation until favourable conditions were attained for insertion of the ProSeal LMA device. Additional boluses of $0.5 \mathrm{mg} \mathrm{kg}^{-1}$ propofol were given as and when required until an adequate level of anaesthesia was achieved for placement of device. Allocation of patients into groups was then done via randomisation method by opening a sealed envelope (by different anaesthetist) into three groups of 42 each.

1. Group SC $(n=42)$ : PLMA was inserted using Suction Catheter. (Fig.1)

2. Group I $(n=42)$ : PLMA was inserted using Introducer tool.

3. Group D $(n=42)$ : PLMA was inserted using Digital technique

A PLMA of appropriate size was chosen according to individual patient's weight and lubricated with a clear water-based jelly. The PLMA was then inserted using different techniques according to the group allocated.

In Group SC, the drain tube of the PLMA was primed with a lubricated $\mathrm{SC}$ with its straight end first, leaving approximately $4-5 \mathrm{~cm}$ bent portion protruding from the proximal end (for the assistant to grip) and the maximum length protruding from the distal end (for the anaesthetist to manipulate). The SC-guided insertion technique involved the following steps:

1. Under gentle laryngoscopic guidance, the distal portion of the $\mathrm{SC}$ was placed nearly $5 \mathrm{~cm}$ into the oesophagus with the assistant holding the PLMA and the proximal portion of the Suction Catheter
2. The laryngoscope was then removed and the PLMA was railroaded over the SC using digital insertion technique

3. The SC was then removed while the PLMA was held in position.

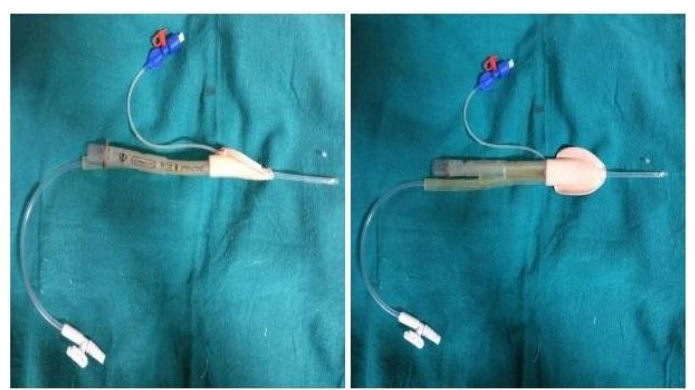

Fig. 1: ProSeal LMA railroaded on Suction Catheter (SC)

In Group D, the index finger was used to press the PLMA into and advanced it around the palatopharyngeal curve. In Group I, The attached Introducer tool (as per standard technique), was used to press the PLMA into and advanced it around the palatopharyngeal curve and the tool was then removed.

All techniques were performed using a midline approach with the patient in the sniffing position' and with the cuff fully deflated, A slight lateral approach was performed for any tactile resistance felt at the back of the mouth. After PLMA insertion, the cuff was inflated with air as per recommendations. Correct placement of the ProSeal LMA device was clinically confirmed by absence of any oropharyngeal air leak, gastric or drain tube air leak, a positive suprasternal notch test and an end-tidal carbon dioxide $\left(\mathrm{EtCO}_{2}\right)$ value between 30 to $45 \mathrm{~mm} \mathrm{Hg}$. A well-lubricated appropriate size gastric tube was then inserted through the drain tube and its placement was assessed by suction of fluid or detection of injected air over epigastric area by stethoscope.

Three attempts were allowed before PLMA insertion was considered a failure (as detected by air leaks, negative suprasternal notch test or failed gastric tube insertion) or ineffective ventilation (maximum expired tidal volume $<8 \mathrm{mlkg}^{-1}$, end tidal carbon dioxide more than $45 \mathrm{mmHg}$ ). The time between picking up the laryngoscope or prepared PLMA (by any method) and successful placement was recorded as "insertion time". If insertion failed even after two attempts, a single attempt with each of the other two techniques in random order was performed. The cause of failed insertion was analysed and documented.

\section{Parameters assessed}

Ease of insertion of the PLMA was assessed by time to insertion and number of attempts tried to achieve effective ventilation along with number of cases in which lateral approach was attempted. 
Hemodynamic response to PLMA placement was assessed using the following parameters: PR, SBP, DBP and MAP.

These parameters were recorded immediately preinsertion $(0 \mathrm{~min})$ and every minute for ten minutes after PLMA insertion. During the anaesthetic procedure, any episode of saturation $\left(\mathrm{SpO}_{2}\right)$ going down below 95\%, related to procedure was observed and recorded in all the groups. Any blood staining on the Suction Catheter, laryngoscope, introducer tool or the PLMA was noted at the time of removal of device. The external and internal oral cavity was inspected for the evidence of trauma related to technique.

Patients and their parents were asked to answer a structured interview 6-24 hr after surgery. They were asked about any complaints of sore throat, dysphonia (voice change) and dysphagia (difficulty in swallowing), hoarseness or change in pattern of crying or swallowing (in case of small children). Symptoms were than graded by the patients or parents as mild, moderate or severe.

Sample size was based upon a projected difference of $15 \%$ between the three groups with respect to the first attempt success rate, a type I error of 0.05 and a power of 0.8 . The continuous data was analyzed with parametric tests like ANOVA, student T-test (paired and unpaired) and non-parametric tests like Kruskal Wallis test, Wilcoxon Mann Whitney test, Wilcoxon signed rank sum test and categorical data was analyzed with the $\chi^{2}$ test or Fischer's exact test. Parametric data was recorded as mean $\pm \mathrm{SD}$ or SE (mean). $\mathrm{P}$ value < 0.05 was taken as significant

\section{Results}

The three groups were similar with respect to their demographic profiles, ASA grading and Mallampati class.

In Group SC, PLMA device was successfully inserted after a single attempt in $41(97.6 \%)$ patients, but PLMA was insertion failed in 5 patients in Group I (11.9\%) and 6 patients in Group D (14.3\% )even after two attempts, and this difference was statistically significant $(\mathrm{p}=0.044)$ (Table 1$)$.

No case in Group SC required the lateral approach, whereas $2(4.8 \%)$ and $3(7.1 \%)$ required the lateral approach for successful insertion in Group I and Group $\mathrm{D}$ each $(\mathrm{p}=0.233)$ (Table 1$)$.

The total insertion of PLMA varied from 19 to 46 seconds in Group S, 17 to 76 seconds in Group I and 18-64 seconds in Group D, The mean time of insertion was $22.7 \pm 4.02$ seconds in Group SC, $31.14 \pm 17.57$ seconds in Group I and 27.1 \pm 12.95 seconds in Group D $(\mathrm{p}<0.011)($ Table 1).

There was no hemodynamic stress response to PLMA insertion in any of the three techniques. Rather, there was a transient fall in the heart rate and blood pressure, which could be due to the pharmacological agents like propofol used for induction of anaesthesia. (Fig. 2-5)

There was no incidence of trauma or blood staining in the Suction Catheter guided PLMA placement whereas there was significantly higher incidence of trauma with the two conventional techniques. Blood staining of the gadgets with the two conventional techniques however was relatively insignificant. There was no difference in postoperative airway morbidity among the groups (Table 1).

Table 1: Demographic Profile, Airway Assessment Data and comparison of PLMA Insertion techniques

\begin{tabular}{|c|c|c|c|c|}
\hline 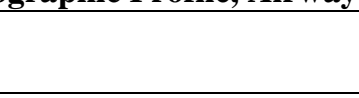 & $\begin{array}{l}\text { Group SC } \\
(n=42)\end{array}$ & $\begin{array}{l}\text { Group I } \\
(n=42)\end{array}$ & $\begin{array}{l}\text { Group D } \\
(n=42)\end{array}$ & p value \\
\hline Age (years) & $6.44 \pm 3.37$ & $5.84 \pm 3.38$ & $5.40 \pm 3.10$ & 0.354 \\
\hline $\operatorname{Sex}(M: F)(n)$ & $33: 9$ & $32: 10$ & $32: 10$ & 0.956 \\
\hline Weight $(\mathrm{kg})$ & $22.70 \pm 8.89$ & $21.56 \pm 9.04$ & $21.90 \pm 9.02$ & 0.837 \\
\hline ASA (I:II) (n) & $40: 2$ & $39: 3$ & $39: 3$ & 0.860 \\
\hline $\begin{array}{l}\text { No. of Attempts } \\
(1: 2: 3: 4)(\mathrm{n})\end{array}$ & $41: 1: 0: 0$ & $33: 4: 4: 1$ & $32: 4: 5: 1$ & \\
\hline $\begin{array}{l}\text { No. of cases req. } \\
\text { alternate technique (n) }\end{array}$ & 0 & 1 & 1 & 0.125 \\
\hline $\begin{array}{l}\text { Successful PLMA } \\
\text { placement in } 1^{\text {st }} \text { attempt }\end{array}$ & 41 & 33 & 32 & $0.013^{*}$ \\
\hline $\begin{array}{l}\text { Successful placement } \\
\text { after } 2 \text { attempts }\end{array}$ & 42 & 37 & 36 & $0.046^{*}$ \\
\hline $\begin{array}{l}\text { Successful placement } \\
\text { after 3attempts }\end{array}$ & 42 & 41 & 41 & 0.602 \\
\hline $\begin{array}{l}\text { Lat. approach of } \\
\text { insertion (n) }\end{array}$ & 0 & 2 & 3 & 0.233 \\
\hline $\begin{array}{l}\text { Total insertion time } \\
\text { (sec.) }\end{array}$ & $22.57 \pm 4.02$ & $31.14 \pm 17.57$ & $27.10 \pm 12.95$ & $0.011 * \$$ \\
\hline
\end{tabular}




\begin{tabular}{|l|c|c|c|c|}
\hline $\begin{array}{l}\text { Leak pressure }(\mathrm{cm} \text { of } \\
\text { H2o) }\end{array}$ & $26.86 \pm 2.18$ & $26.69 \pm 1.96$ & $26.98 \pm 2.17$ & 0.824 \\
\hline Blood staining $(\mathrm{n})$ & 0 & 2 & 4 & 0.122 \\
\hline Trauma $(\mathrm{n})$ & 0 & 3 & 7 & $0.018^{*}$ \\
\hline
\end{tabular}

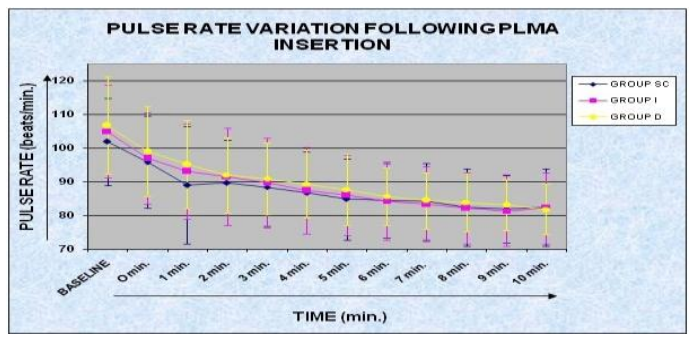

Fig. 2 Comparison of Pulse rate following PLMA insertion in all three groups

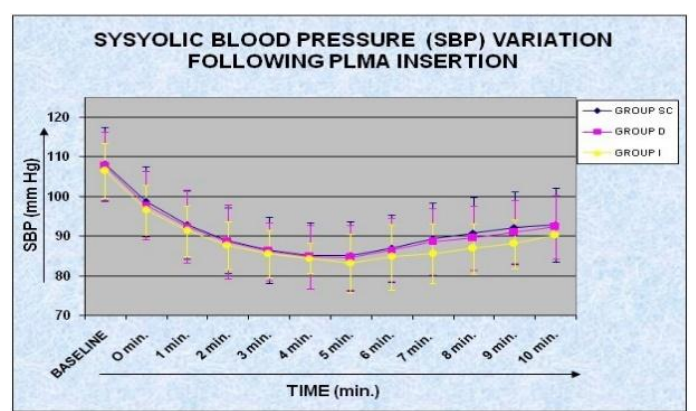

Fig. 3: Comparison of Systolic BP following PLMA insertion in all three groups

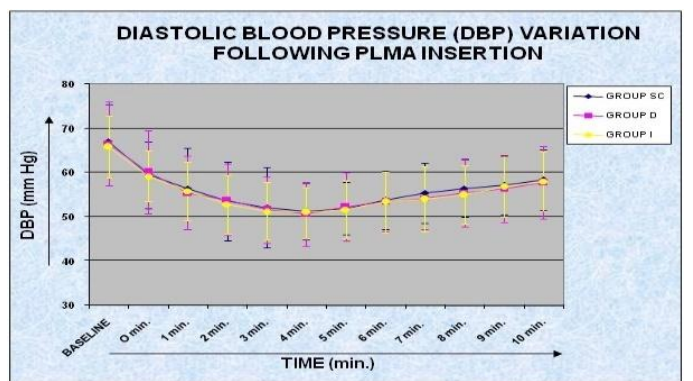

Fig. 4: Comparison of Diastolic BP following PLMA insertion in all three groups

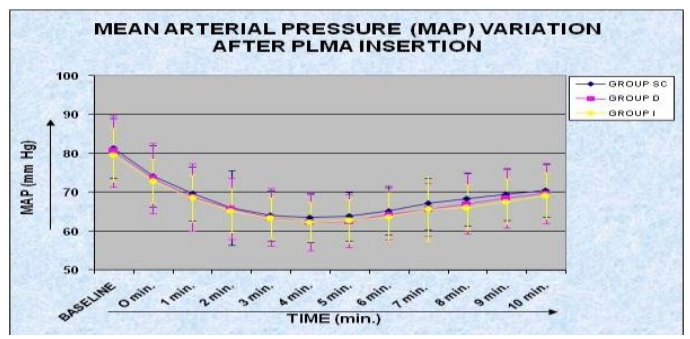

Fig. 5: Comparison of Mean arterial pressure following PLMA insertion in all three groups

\section{Discussion}

The ProSeal LMA is the most ingenious and complex specialized LMA device with a primary goal to provide better ventilation and protection against regurgitation and gastric insufflation. ${ }^{3}$ The PLMA as a supraglottic device broadens the applicability of airway devices in clinical situations where better seal, airway protection and access to oesophagus are required. ProSeal LMA has gained wide popularity in paediatric anaesthesia since its introduction into clinical practice in 1990. Although it was initially used as a replacement for the face mask, it is now used in areas where the endotracheal tube was formerly used. ${ }^{9}$ Various authors have proven that the paediatric PLMA forms a more effective seal than the classic LMA with additional advantage of gastric tube placement. ${ }^{9-11}$ The unique design of the paediatric PLMA (absence of dorsal cuff) further seems to be beneficial as indicated by a higher first attempt placement success rate with higher rate of anatomically correct position in comparison to classic LMA.

In our study, we compared the ease of insertion of a PLMA device based on the insertion time, number of insertion attempts to achieve effective ventilation and number of cases requiring lateral approach of insertion. The three groups were comparable according to demographic profiles i.e. in the age and sex distribution, the height and weight distribution and the ASA grades. According to results of our study, insertion of PLMA device was successful in $97.6 \%$ in Group SC, 78.6\% in Group I, and 76.2\% in Group D, and the difference was statistically significant. Our results were consistent with that of Agudo et $\mathrm{al}^{8}$ found that insertion was more frequently successful (SC, 97\%; digital, $89 \%$ ) with the SC guided technique. In our study, there was statistically insignificant difference in the insertion success rate between the Introducer Tool and Digital technique. Evans et $\mathrm{al}^{13}$ in a descriptive trial of 300 cases reported similarly no difference in the insertion success rates of Introducer Tool and Digital techniques. But Brimacombe et al. ${ }^{12}$ reported that first time success rate with the introducer tool was higher as compared to digital method. According to him, introducer used lesser area as compared to digital method, also it directed cuff over the oropharyngeal inlet and facilitated good depth of insertion. Nevertheless the study size carried by him was small as compared to the previous ones. ${ }^{13-14}$

However, there is a paucity of data in paediatric patients comparing directly introducer and digital technique, as most of the authors have considered success and failure of PLMA insertion in paediatric patients to be similar as they are in adults. ${ }^{7}$

We found that the PLMA insertion successes after two attempts in our groups were: $100 \%$ in Group SC, $88 \%$ in Group I, and $85.7 \%$ in Group D, and the difference being statistically significant. While after three attempts success rate was Group SC, 100\%; 
Group I 97.6\% and Group D, 97.6\%, the difference being statistically significant. These results were in consistent to that of Brimacombe et al. (IT, 98\%; digital, 99\%;) and Agudo et al. (SC, 100\%; digital, 98\%;) who found that the difference in the overall success rates was statistically not significant. ${ }^{8}$ with repeated attempts the success rate of insertion of the introducer tool and the digital techniques might increase but it also increases the chances of hypoxia because of the increased time in securing an airway.

Lateral approach was required in $2(4.8 \%)$ patients in Introducer Tool group and $3(7.1 \%)$ patients in Digital technique groups but not even in a single case in the SC guided insertion group. This was statistically significant only in case when digital technique group was compared with SC guided technique and was in consistent to the findings of Agudo et al. who reported a statistically significant difference in patients requiring lateral approach in the SC guided insertion group $(0 \%)$ and in the other digital $12 \%$ technique in adults.

Successful placement of PLMA device took lesser time in SC guided technique $(22.57 \pm 4.021 \mathrm{~s})$ as compared to other two groups (Group I, $31.14 \pm 17.57 \mathrm{~s}$; Group D, 27.10 $\pm 12.95 \mathrm{~s}$ ). Our findings were similar to Brimacombe et al. ${ }^{1}$ and Agudo et al. ${ }^{8}$ This may be because of first time higher success rate in PLMA placement by SC guided technique was $(97.6 \%)$ and more than a single attempt was required in many cases for successful PLMA insertion in conventional techniques. The other reason may be, because lateral approach was required in few cases in group I and D whereas not even a single patient in the SC guided group required so. Moreover the finger technique is less successful as the larger cuff is more difficult to place in the mouth, leaving less space for the index finger manipulation and is more likely to fold over. ${ }^{12}$ These factors may be responsible for increased time taken in the other two groups. Thirdly, insertion of PLMA was done via gentle laryngoscopy in SC guided group which might have attributed to the shorter time taken to insert the PLMA.

In the SC guided PLMA insertion group there was an appreciable decrease in Pulse Rate, Systolic Blood Pressure, Diastolic Blood Pressure and Mean Blood Pressure at $0,1,2,3,4,5,6,7,8,9$ and $10 \mathrm{~min}$. of insertion.(Fig. 2-5) This is in consistent to Lopez-Gil et al., ${ }^{7}$ who reported no increase rather fall in the heart rate or blood pressure during laryngoscope guided Gum Elastic Bougie guided insertion (similar to suction catheter guided technique) in a study of 60 children. Thus in spite of using laryngoscope in the SC guided PLMA insertion group, there was no hemodynamic stress response unlike the laryngoscopy routinely done for intubation. This could be because in this technique we applied just enough force to visualize the posterior epiglottis. The time duration of laryngoscopy while inserting the $\mathrm{SC}$ is also shorter than while intubation, as posterior glottis is visible in almost all cases whereas anterior glottis is less commonly visible, thereby increasing the duration of laryngoscopy. It has been found that duration of direct laryngoscopy is important for limiting the pressor response and its duration should not exceed 15 seconds [15] to minimize the increase in systemic blood pressure. Laryngoscopy is necessary for insertion of SC so as to visualize the tip of SC entering the oesophagus. This avoids unnecessary pharyngeal trauma by the SC (when insertion tried blindly) and accidental insertion of the tip of SC in glottis area, thereby avoiding the malpositioning of the PLMA.

In the introducer tool and digital techniques also, there was no hemodynamic stress response (increase heart rate, increase blood pressure) to PLMA placement (rather a fall in the hemodynamic parameters was noted which could be due to the effect of induction agents like Propofol used in our study). These results were consistent with Evans et al. ${ }^{13}$ who in a descriptive study of 300 adults found that there were no haemodynamic response to PLMA insertion, with a further small decrease in heart rate $5 \mathrm{~min}$ after insertion and significant reduction in mean arterial pressure at 1 and 5 min. interval after insertion. Lopez-Giletal. ${ }^{7}$ have also reported fall in post insertion values of mean arterial pressure and heart rate in comparison to pre insertion values in a study of 60 children, where PLMA was inserted by digital technique.

Hypoxia was not reported in any our cases in all the three groups. There was not a single case of blood staining of the SC, laryngoscope or the PLMA in the SC guided PLMA insertion group. Aguado et al. ${ }^{8}$ reported blood stains on device in $8 \%$ cases, in whom PLMA was inserted using the SC technique. However they found no blood on the suction catheter on its removal suggesting no oesophageal mucosal trauma occur following passage of the suction catheter. There could have been several reasons for this. The laryngoscopy required is gentle to visualize the posterior glottis; ${ }^{6}$ and suction catheter is an atraumatic, yet is stiff enough ${ }^{8}$ to be able to insert easily; and railroading the PLMA over it avoids impaction at the back of the mouth. The SC guided technique also reduces the total number of attempts required for successful PLMA insertion. Although in the other two techniques there were cases of blood staining (4 cases, 9.5\% of blood staining of PLMA in group D and 2 case, $4.8 \%$ of blood staining of Introducer tool in group I); these results were statistically not significant. Aguado et al. ${ }^{8}$ reported staining of PLMA device with blood in $5 \%(3 / 60)$ of their patients with digital technique. While, Kanthed et al. ${ }^{16}$ reported blood staining in $8 \%$ (4/50) of patients using the introducer tool technique. The staining of blood might be because of impaction of device at the back of mouth and during intraoral manipulations (e.g. using the lateral approach) required in insertion of the PLMA. 


\section{Conclusion}

SC-guided ProSeal LMA insertion in children was an easier, more successful, faster, and a relatively atraumatic technique which also ensures correct placement of the PLMA in the hypopharynx as compared to the routine conventional PLMA insertion techniques. There was also no hemodynamic pressor response to PLMA insertion using any of the three techniques.

\section{References}

1. Refe Lopez-Gil M, Brimacombe J. The Proseal laryngeal mask airway in children. Paediatr Anaesth 2005;15:22934.

2. LMA. LMA ProSealTM Instruction manual, revised. San Diego: LMA North America Inc 2005.

3. Brimacombe J. ProSeal LMA for ventilation and airway protection. In: Laryngeal Mask Anesthesia. Principles and Practice. London: WB Saunders; 2005,505-38.

4. Brimacombe J, Keller C, Vosoba Judd D. Gum elastic bougie-guided insertion of the ProSealTM laryngeal mask airway is superior to the digital and introducer tool techniques. Anesthesiology 2004;100:25-9.

5. Brimacombe J, Keller C. Gum elastic bougie-guided insertion of the ProSealTM laryngeal mask airway. Anaesth Intensive Care 2004;32:681-4.

6. Howarth A, Brimacombe J, Keller C. Gum elastic bougie-guided insertion of the ProSeal laryngeal mask airway. A new technique. Anaesth Intensive Care 2002;30:624-7.

7. Lopez-Gil M, Brimacombe J, Barragan L, Keller C. Bougie guided insertion of the proseal laryngeal mask airway has higher first attempt success rate than the digital technique in children. Br J Anaesth 2006;92:23841.

8. Aguado-Garcia R, Vinoles J, Brimacombe J, Vivo M. Suction catheter guided insertion of the proseal laryngel mask airway is superior to the digital technique. Can J Anesth 2006;53:398-403.

9. Goldmann K, Jakob C. Size 2 ProSeal $^{\mathrm{TM}}$ laryngeal mask airway: a randomized, crossover investigation with the standard laryngeal mask airway in paediatric patients. $\mathrm{Br}$ J Anesth 2005;94:385-9.

10. Shimbori H, Ono K, Miwa T, Morimura N. comparison of the LMA-Proseal and LMA Classic in children. Br J Anesth 2004;93:528-31.

11. Keller C, Brimacombe J. Mucosal pressure and oropharyngeal leak pressure with the Proseal vs. CLMA. Br J Anesth 2000;85:262-6.

12. Brimacombe J, Keller C. The proseal laryngeal mask airway: A randomized, crossover study with the standard laryngeal mask airway in paralyzed, anesthetized patients. Anesthesiology 2000;93:104-9.

13. Evans N.R., Gardner S.V., James M.F.M. etal. The ProSeal laryngeal mask: results of a descriptive trial with experience of 300 cases. Br J Anesth 2002;88(4):534-9.

14. Cook TM, Nolan JP, Verghese C, et al. Randomized crossover comparison of the proseal with the classic laryngeal mask airway in unparalyzed anesthetized patients. Br J Anaesth 2002;88:527-33.

15. Stoelting RK, Dierdorf SF. Systemic Hypertension. Anesthesia and Co-Existing Disease. 4 th edn.102.

16. Kanthed P Sharma B, Sood J, Kumra V. Comparison of LMA-ProSeal $^{\mathrm{TM}}$ with LMA Classic ${ }^{\mathrm{TM}}$ in Anaesthetised Paralysed Children. Indian Journal of Anaesthesia 2008;52:44-8. 